\title{
The TAL Suction Retractor: A Simple Technique for Visualising the Transverse Acetabular Ligament (TAL) During Total Hip Replacement (THR) Surgery
}

\author{
Murphy Ben, MB BCh BAO, MCh, MRCSI*, Pollock Roisin, $M B B C h$ BAO, Ramchurn Sumant, \\ $M D$ and Hurson Conor, MB BCh BAO, AFRCSI, MCh, FRCSI
}

Department of Trauma \& Orthopaedic Surgery, St Vincent's University Hospital, Elm Park, Ireland

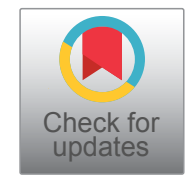

\section{Keywords}

Transverse acetabular ligament, Total hip replacement, THR, Hip, Acetabular, Suction, Retractor

\section{Introduction}

The transverse acetabular ligament (TAL) is an anatomical landmark that is of particular use during primary total hip replacements (THR). It can be used to control acetabular depth, height and version [1]. Visualisation is important to aid the orientation of the acetabular cup and it is a structure that can be identified in virtually every primary hip replacement surgery [2]. A study of 1,000 consecutive primary THRs identified and exposed the TAL in $99.7 \%$ of cases [2].

The advantages posed by the TAL are that it is independent of patient positioning, cup version can be individualised to the patient and it is an easy-to-teach technique [3]. Alignment of the acetabular component parallel to the TAL as a soft tissue landmark can lead to reduced malpositioning of the acetabular component in THR according to the traditional safe zones thus reducing the risk of dislocation [4].

\section{Technique}

Having highlighted the importance of directly visualising the TAL to aid with orientation of the acetabular component in THR, we now describe a very simple technique for maintaining visualisation during acetabular preparation, template trial and cup implantation.

A thoracic suction catheter is taped to the distal end of a blunt Hohmann's retractor with two steristrips wrapping around the catheter and retractor leaving approximately $1-1.5 \mathrm{~cm}$ of retractor exposed (Figure 1). Our preference is for a thoracic suction catheter with side holes and a proximal end which allows direct connection with the tip of the Yankauer suction.

The blunt Hohmann's retractor is used as the inferior acetabular retractor in the usual way, placed inferior to the transverse acetabular ligament, resting on the posterior ischi- al surface. The catheter suction tip should lie just under the TAL, in the most dependant point of the wound (Figure 2). The TAL suction catheter can be used continuously or intermittently to facilitate visualisation of the TAL during, reaming, trialling and acetabular cup placement.

The surgeon's assistant, who would ordinarily maintain suction, may now assist the primary surgeon with other intra-operative tasks.

The TAL suction retractor allows for a largely bloodless field that permits direct visualisation of TAL throughout acetabular cup trial and placement (Figure 3).

\section{Conclusion}

The transverse acetabular ligament (TAL) suction retractor is a simple improvised surgical tool that makes it easier to visualise the TAL during acetabular preparation in total hip replacements. The TAL suction retractor provides suction in the most dependant point of the surgical wound, preventing unnecessary movement and facilitates a clutter-free surgical field leading to more streamlined and efficient surgery with potential reduced operative times.

*Corresponding author: Ben Murphy, MB BCh BAO, MCh, MRCSI, Department of Trauma \& Orthopaedic Surgery, St Vincent's University Hospital, Elm Park, Dublin, Republic of Ireland

Accepted: February 10, 2021

Published online: February 12, 2021

Citation: Ben M, Roisin P, Sumant R, et al. (2021) The TAL Suction Retractor: A Simple Technique for Visualising the Transverse Acetabular Ligament (TAL) During Total Hip Replacement (THR) Surgery. J Orthop Surg Tech 4(1):244-245 
Citation: Ben M, Roisin P, Sumant R, et al. (2021) The TAL Suction Retractor: A Simple Technique for Visualising the Transverse Acetabular Ligament (TAL) During Total Hip Replacement (THR) Surgery. J Orthop Surg Tech 4(1):244-245

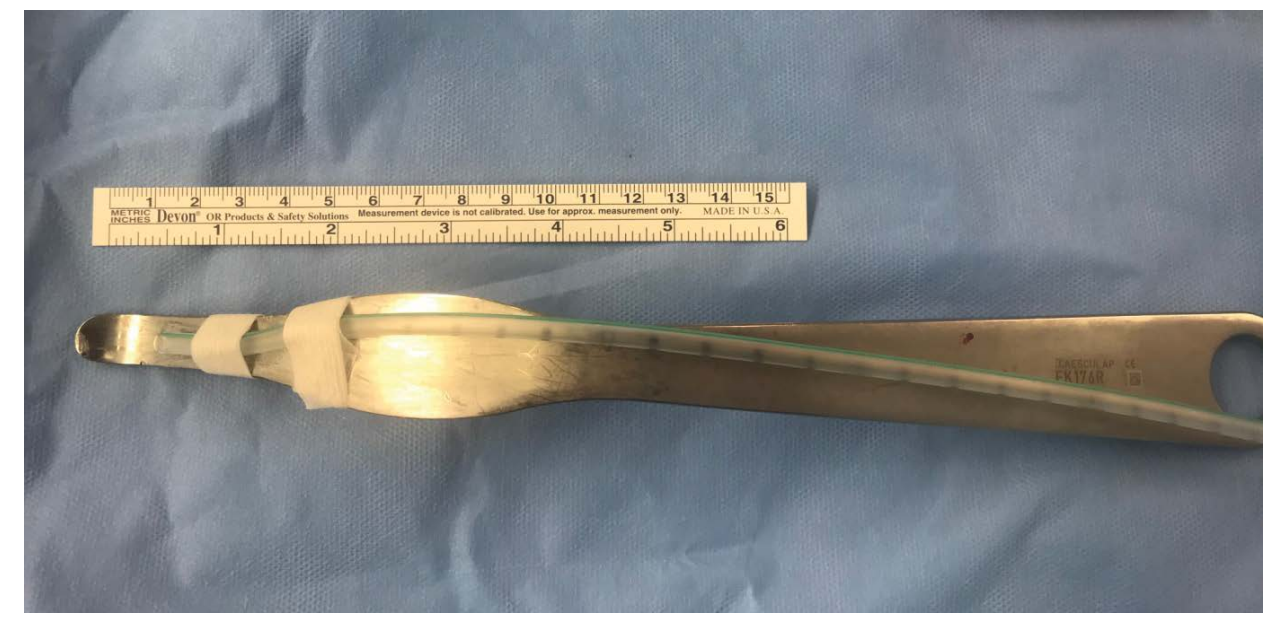

Figure 1: Blunt Hohmann's retractor with a thoracic suction catheter steri-stripped to the distal end.

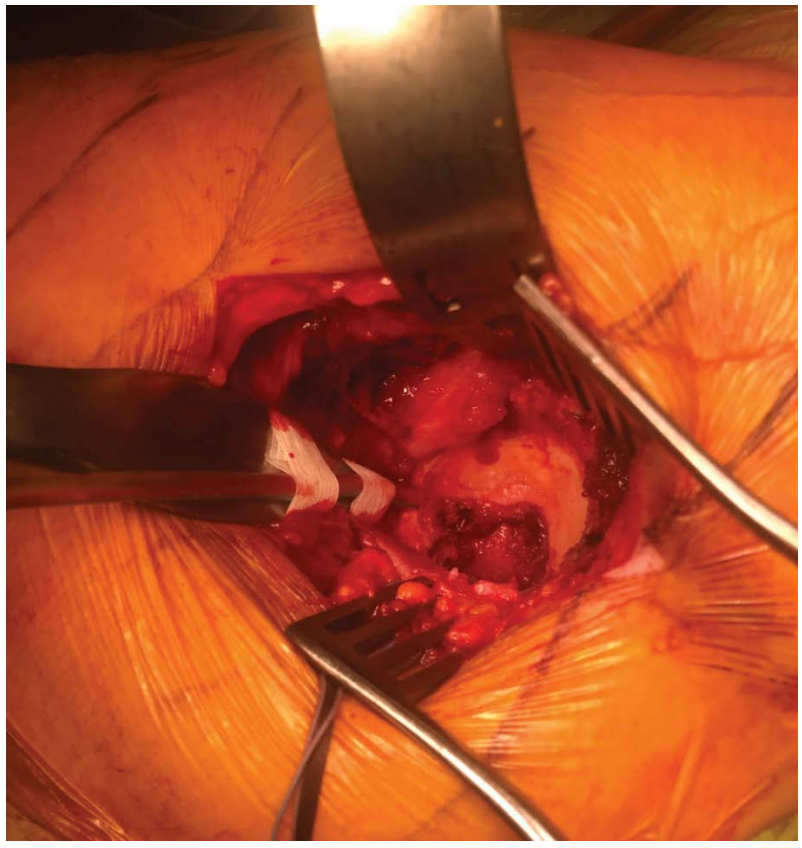

Figure 2: TAL suction retractor in-situ after acetabular exposure with clear visualisation of the transverse acetabular ligament.

\section{Conflict of Interest}

None.

\section{References}

1. Beverland DE, O Neill CKJ, Rutherford M, et al. (2016) Placement of the acetabular component. The Bone \& Joint Journal 98: $37-$ 43.

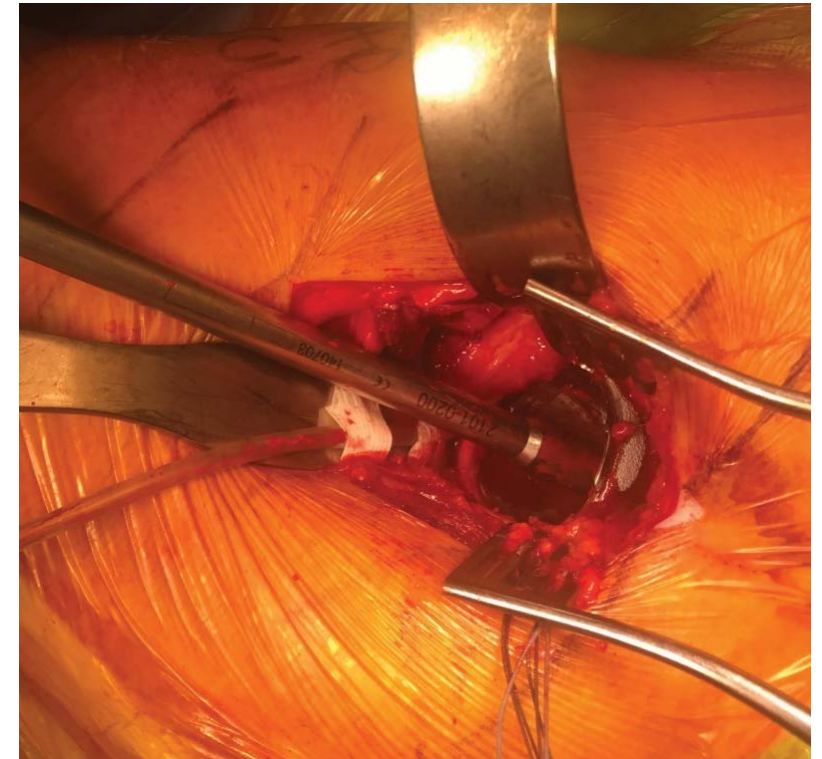

Figure 3: The TAL suction catheter providing visualisation of the TAL during all stages of definitive acetabular cup placement.

2. Archbold HAP, Mockford B, Molloy D, et al. (2006) The transverse acetabular ligament: An aid to orientation of the acetabular component during primary total hip replacement: A preliminary study of 1000 cases investigating postoperative stability. The Journal of Bone and Joint Surgery 88: 883-886.

3. Beverland D (2010) The transverse acetabular ligament: Optimizing version. Orthopedics 33: 631.

4. Kalteis T, Sendtner E, Beverland D, et al. (2011) The role of the transverse acetabular ligament for acetabular component orientation in total hip replacement. The Journal of Bone and Joint Surgery. British volume, 93: 1021-1026.

DOI: $10.36959 / 453 / 555$ 